\section{Comparación de 3 modos de ventilación mecánica en colecistectomía laparoscópica}

\author{
Comparison of 3 mechanical ventilation modes \\ in laparoscopic cholecystectomy
}

\author{
Dra. Lizbeth Cañas-Lucero,* Dr. Héctor Carrero-Soto, ${ }^{\ddagger}$ Dra. Alinne Lemus-Cázares, ${ }^{\S}$ \\ Dr. José Luis Limón-Aguilar, ${ }^{\natural}$ Dra. Patricia Vázquez-Estrada, ${ }^{\ddagger}$ \\ Dra. Yolanda Valera-Rodríguez, ${ }^{\ddagger}$ Dra. María Elena Pinto-Segurall
}

Citar como: Cañas-Lucero L, Carrero-Soto H, Lemus-Cázares A, Limón-Aguilar JL, Vázquez-Estrada P, Valera-Rodríguez Y, et al. Comparación de 3 modos de ventilación mecánica en colecistectomía laparoscópica. Rev Mex Anestesiol. 2021; 44 (3): 178-183. https://dx.doi.org/10.35366/99664

\begin{abstract}
RESUMEN. El modo ventilatorio asistido durante un procedimiento laparoscópico aún es controversial. Objetivo: Comparar la dinámica ventilatoria según el modo de ventilación asistida: por ventilación controlada por volumen (VCV), controlada por presión (VCP) o por presión con garantía de volumen (VCP-GV), en anestesia para colecistectomía laparoscópica. Material y métodos: 21 pacientes adultos manejados con una de las tres modalidades (7 por grupo). Se analizó durante el procedimiento (a la intubación, la insuflación de $\mathrm{CO}_{2}$ intraabdominal y resolución), su frecuencia respiratoria, el volumen espiratorio, $\mathrm{CO}_{2}$ al final de la espiración $\left(\mathrm{EtCO}_{2}\right)$, presión pico vía aérea, presión media pulmonar, distensibilidad, saturación de oxígeno y volumen minuto respiratorio. Resultados: Posterior a la intubación, no hubo diferencias clínicas importantes en las mediciones entre los modos de ventilación. A la insuflación los pacientes con modo VCP incrementaron su frecuencia respiratoria, pero conservaron su presión pico; contra aquéllos en modo VCV y VCP-GV que incrementaron su presión pico con escasa reducción de su frecuencia respiratoria. Las otras variables no se modificaron durante el procedimiento y no hubo diferencias entre los modos ventilatorios. Conclusión: Los tres modos de ventilación permitieron un buen control ventilatorio con pocas diferencias respecto a parámetros basales, pero sugerimos que el modo VCP previene mejor los aumentos en la presión pico.
\end{abstract}

ABSTRACT. The assisted ventilatory mode during a laparoscopic procedure is still controversial. Objective: To compare ventilatory dynamics according to the assisted ventilation mode: by volume-controlled ventilation (VCV), pressure-controlled (PCV) or by pressure with volume guarantee (PVC-VG), in general anesthesia for laparoscopic cholecystectomy. Material and methods: 21 adult patients managed with one of the three modalities (seven per group). Their respiratory rate, minute expiratory volume, end tidal $\mathrm{CO}_{2}\left(\mathrm{EtCO}_{2}\right)$, peak airway pressure, mean pulmonary pressure, compliance, oxygen saturation and minute respiratory volume were analyzed during the procedure (at intubation, abdominal $\mathrm{CO}_{2}$ insufflation and resolution). Results: After intubation there were no clinical differences in measurements between ventilation modes. On insufflation, patients with PCV mode increased their respiratory rate, but kept their peak pressure; against those in VCV and PCV-VG mode who increased their peak pressure with little reduction in their respiratory rate. The other variables were not modified during the procedure and there were no differences between the ventilatory modes. Conclusion: The three modes of ventilation allowed a good ventilatory control, but we suggest the PCV since it prevents an increase in peak pressure.

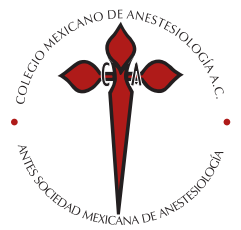

Palabras clave:

Ventilación mecánica, colecistectomía laparoscópica, modo ventilatorio, barotrauma, anestesia general, atelectasias.

Keywords:

Mechanical ventilation, laparoscopic cholecystectomy, ventilatory mode, barotrauma, general anesthesia, atelectasis.

* Residencia de Anestesiología, Facultad Mexicana de Medicina de la Universidad La Salle. Ciudad de México, México.

${ }^{\ddagger}$ Médico adscrito al Servicio de Anestesiología, México.

$\S$ Médico adscrito a la Unidad de Cuidados Intensivos, Hospital Ángeles Clínica Londres. México. " Médico adscrito al Servicio de Cirugía General.

॥ Profesora Titular del Curso de Anestesiología, Facultad Mexicana de Medicina de la Universidad La Salle. Ciudad de México, México.

Correspondencia:

Dra. Lizbeth Cañas-Lucero

Durango Núm. 50,

Col. Roma Norte, 06700, alcaldía Cuauhtémoc, CDMX.

E-mail: lizbethclmx@hotmail.com

Recibido: 03-07-2020

Aceptado:09-10-2020

\section{INTRODUCCIÓN}

L a mecánica respiratoria es una compleja interacción entre la pared torácica y los pulmones ${ }^{(1)}$, pero un buen manejo intraoperatorio permite una ventilación a través de un flujo de aire con presión positiva y con un volumen corriente adecuado ${ }^{(1)}$.

En las cirugías laparoscópicas ante la insuflación de dióxido de carbono $\left(\mathrm{CO}_{2}\right)^{(2)}$ abdominal es posible la afectación de la función pulmonar. Esto se debe al incremento de la presión intraabdominal (PIA) que desplaza el diafragma en dirección cefálica, exacerbado por la posición de Trendelenburg ${ }^{(2)}$. Las cuatro complicaciones pulmonares asociadas a la insuflación abdominal incluyen: atelectasias ${ }^{(3)}$, hipoxemia, reducción de la distensibilidad pulmonar y enfisema subcutáneo. La hipercarbia además puede causar vasodilatación sistémica, arritmias, depresión miocárdica y exacerbación de la hipertensión pulmonar ${ }^{(2)}$.

El principal objetivo de la ventilación mecánica intraoperatoria es optimizar el intercambio gaseoso, y minimizar el daño 
pulmonar inducido por la misma ${ }^{(4)}$, con ello, se logra mantener una adecuada tensión de oxígeno tisular $\left(\mathrm{PtisO}_{2}\right)$. Para lograrlo, se requieren condiciones hemodinámicas adecuadas y optimización del intercambio de gases logrado con una ventilación adecuada ${ }^{(5)}$.

En la actualidad, para lograr los objetivos ventilatorios se han propuesto diferentes métodos: el ventilatorio controlado por presión (VCP), el controlado por volumen (VCV) y el controlado por presión con garantía de volumen (VCP-GV).

En el modo VCP con respecto al VCV entrega la mayor parte del volumen corriente tempranamente durante la fase inspiratoria ${ }^{(5)}$, aumenta más el tiempo de permanencia alveolar del volumen corriente y produce presiones de la vía aérea más altas; todo ello mejora el intercambio gaseoso ${ }^{(4)}$. El principal inconveniente de VCP es su dependencia en la distensibilidad como de la resistencia del sistema respiratorio. En consecuencia, cualquier condición quirúrgica que disminuya el cumplimiento o aumente la resistencia del sistema respiratorio disminuirá la ventilación alveolar ${ }^{(6)}$.

El modo VCV es ampliamente usado en anestesia general dado que garantiza un volumen corriente predeterminado. Sin embargo, presenta el riesgo de un aumento de la presión en las vías respiratorias cuando cambia la distensibilidad pulmonar. A diferencia del modo VCP que tiene menos riesgo de barotrauma.

Durante el neumoperitoneo (en cirugía laparoscópica), se puede abogar por el modo VCP debido a un aumento significativo en la presión de la vía aérea después de la insuflación de $\mathrm{CO}_{2}$. Sin embargo, aún hay controversia sobre el beneficio en la cirugía laparoscópica ${ }^{(1,7)}$. Es común en la práctica habitual

\begin{tabular}{lccc}
\multicolumn{5}{c}{ Tabla 1: Características de los 21 participantes } \\
según modalidad de ventilación.
\end{tabular}

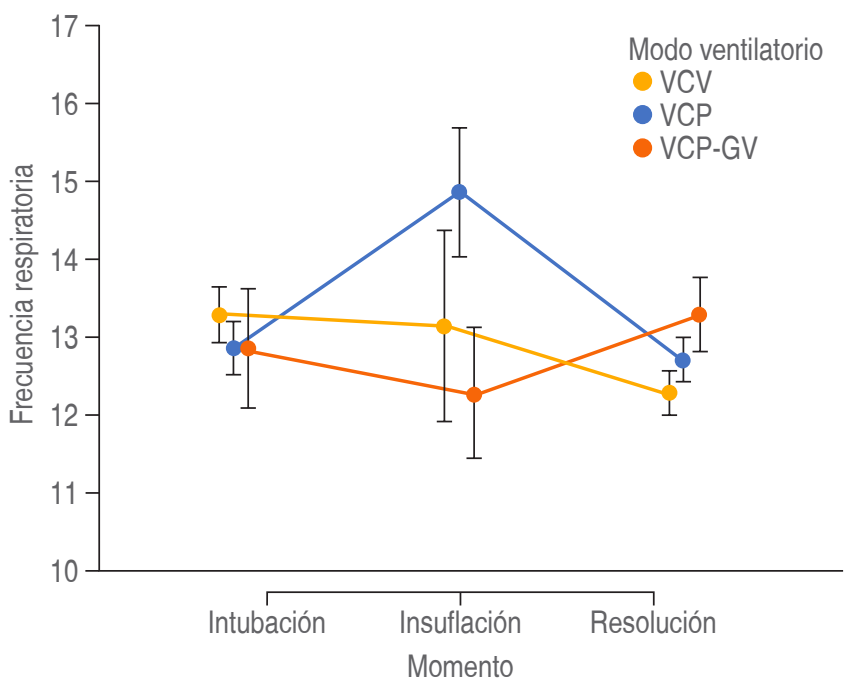

Figura 1: Comportamiento de la frecuencia respiratoria (ciclos por minuto) en los diferentes momentos de evaluación según el modo de ventilación controlada: por volumen (VCV), por presión (VCP) o por presión con garantía de volumen (VCPGV). Los puntos indican la media, las barras errores típicos (1ET). No se encontró efecto del momento $(F=1.18, p=0.32)$, ni por modo ventilatorio $(F=0.49, p=$ $0.62)$, hubo interacción modo-momento para el grupo $\operatorname{VCP}(F=5.7, p=0.04)$.

observar cambios entre los modos cuando ocurre un problema de oxigenación intraoperatoria ${ }^{(4)}$.

La ventilación controlada por presión con garantía de volumen (VCP-GV) es un modo de ventilación bajo control de volumen regulado por presión que comparte características de la VCV y la VCP. La VCP-GV otorga un volumen corriente con una presión inspiratoria constante, utilizando un patrón de flujo desacelerado ${ }^{(1)}$.

Los parámetros del ventilador se ajustan automáticamente con cada respiración del paciente para ofrecer el volumen corriente objetivo sin aumentar las presiones de la vía aérea. Con ello, se disminuye el riesgo de barotrauma ${ }^{(8)}$. A pesar de ello, no se ha establecido la superioridad de un modelo ventilatorio sobre otro.

Un estudio realizado en el 2014 concluyó que el modo controlado por volumen resultaba en una mejor ventilación alveolar ${ }^{(9)}$; sin embargo, estudios más recientes no demostraron diferencia significativa respecto al modo controlado por presión ${ }^{(10,11)}$.

La incidencia del barotrauma pulmonar depende en gran medida de la indicación subyacente para la ventilación mecánica. Varios ensayos y un metaanálisis han estimado la prevalencia entre 0 y 50\%. Los datos más recientes después de la implementación de estrategias de ventilación con protección pulmonar parecen estar más cerca de una incidencia promedio del $10 \%$ entre diferentes poblaciones ${ }^{(12)}$.

Un ensayo aleatorizado de Youn Yi Jo y colaboradores mostró que el modo VCP proporcionó presiones máximas más bajas en las vías respiratorias y mayor distensibilidad dinámica de los pulmones a los 60 y 120 minutos durante la prostatectomía radical 
laparoscópica asistida por robot en la posición de Trendelenburg, con respecto a la VCV en volúmenes corrientes similares ${ }^{(13)}$.

En este trabajo comparamos tres modos de ventilación mecánica (VCP, VCV y VCP-GV) con el objetivo de analizar cuál de ellos mantiene mejor la dinámica ventilatoria basal y reduzca los riesgos de complicaciones postoperatorias pulmonares.

\section{MATERIAL Y MÉTODOS}

Se realizó un ensayo clínico abierto durante el período del 1 de enero al 28 de febrero del 2020 en el Hospital Ángeles Clínica Londres. Para responder a nuestra comparación entre los modos de ventilación, contactamos con los anestesiólogos de nuestra unidad. Sabiendo de antemano la preferencia en el modo de ventilación y de acuerdo con la programación de pacientes que iban a ser sometidos a una colecistectomía laparoscópica electiva, invitamos al paciente e informamos al anestesiólogo nuestra intensión de monitorizar la función respiratoria.

Los pacientes debían cumplir con los siguientes criterios de selección: tener entre 18 y 80 años, no padecer de enfermedades pulmonares (agudas o crónicas) y con la clasificación del estado físico según la Sociedad Americana de Anestesiólogos (ASA) I y II. Se excluyeron aquéllos con antecedentes de procesos infecciosos respiratorios con menos de tres semanas de resolución. Para fines del análisis se eliminarían pacientes en quienes el procedimiento laparoscópico se cambió a uno abierto.

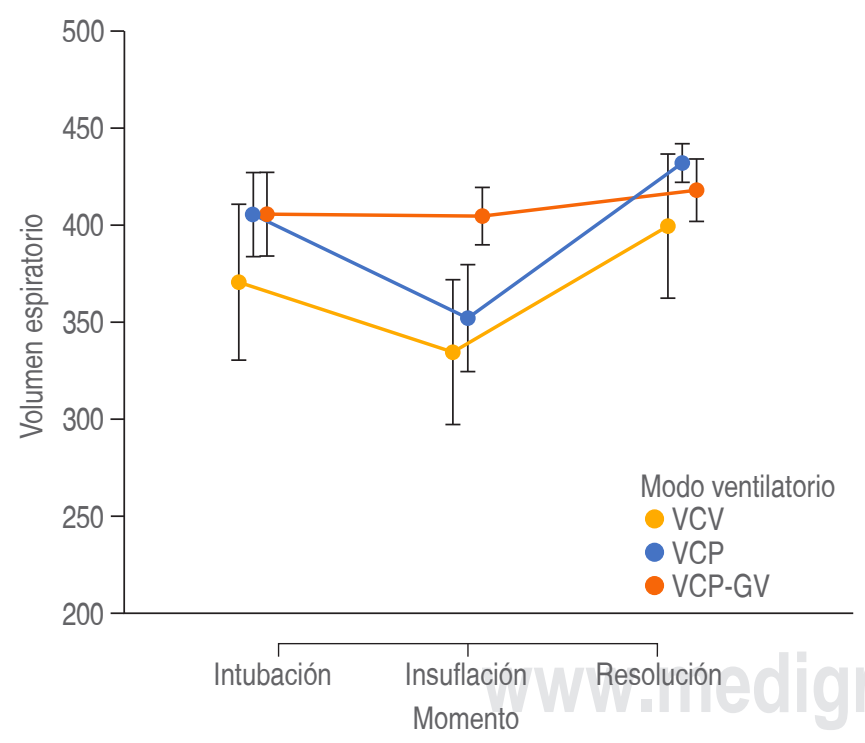

Figura 2: Comportamiento del volumen espiratorio (litros por minuto) en los diferentes momentos de evaluación según el modo de ventilación controlada: por volumen (VCV), por presión (VCP) o por presión con garantía de volumen (VCP-GV). Los puntos indican la media, las barras errores típicos (1ET). Se encontró efecto del momento ( $F=14.6, p<0.001)$, no por el modo ventilatorio $(F=0.69, p=0.52)$, ni interacción modo-momento $(F=2.3, p=0.08)$.

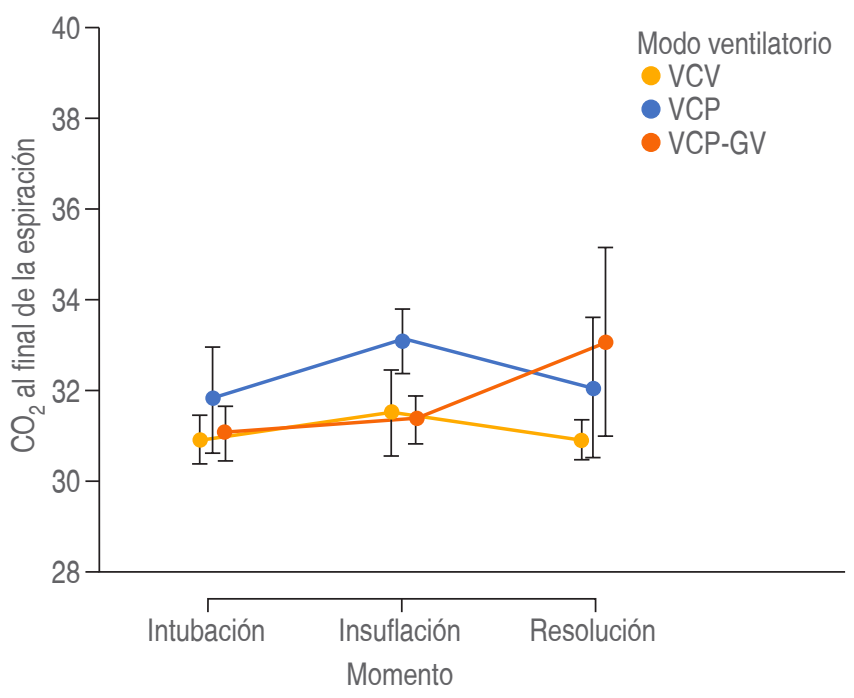

Figura 3: Comportamiento del dióxido de carbono al final de la espiración $(\mathrm{mmHg})$ en los diferentes momentos de evaluación según el modo de ventilación controlada: por volumen (VCV), por presión (VCP) o por presión con garantía de volumen (VCP-GV). Los puntos indican la media, las barras errores típicos (1ET). No se encontró efecto del momento ( $F=$ $0.59, p=0.56)$, ni por el modo ventilatorio ( $F=0.66, p=0.53)$, ni interacción modo-momento $(F=0.64, p=0.64)$.

Una vez establecido el modo ventilatorio (controlado por volumen [VCV], controlado por presión [VCP] o controlado por presión con garantía de volumen [VCP-GV]) seleccionamos a siete participantes por cada modo.

Durante el procedimiento anestésico se colectó información sobre: edad, sexo, peso, talla, índice de masa corporal (IMC), peso predicho $(\mathrm{PBW})^{(14)}$, su clasificación del estado físico de la Sociedad Americana de Anestesiólogos (ASA) y la presencia de comorbilidades (hipertensión arterial sistémica, diabetes mellitus e hipotiroidismo). De las condiciones ventilatorias registradas en tres momentos (después de la intubación y antes de iniciar el neumoperitoneo, 10 minutos después de la insuflación de $\mathrm{CO}_{2}$ intraabdominal, y a la resolución del pneumoperitoneo): frecuencia respiratoria (por minuto), volumen espirado (Vtesp, $\mathrm{mL}$ ), dióxido de carbono al final de la espiración $\left(\mathrm{EtCO}_{2}\right.$, $\mathrm{mmHg}$ ), presión pico en la vía aérea (Ppico, $\mathrm{cmH}_{2} \mathrm{O}$ ), presión media durante el ciclo respiratorio (Pmedia, $\mathrm{cmH}_{2} \mathrm{O}$ ), distensibilidad dinámica (Compl, $\mathrm{cmH}_{2} \mathrm{O}$ ), saturación de oxígeno por pulsioximetría $\left(\mathrm{SpO}_{2}\right.$, \%) y volumen minuto (VM, L/min).

Durante el procedimiento laparoscópico, se aseguró que la presión de insuflación del neumoperitoneo no rebasara los $12 \mathrm{cmH}_{2} \mathrm{O}$. La dosis de bloqueador neuromuscular se tituló para mantener un tren de cuatro (TOF) 0.

El estudio fue aceptado por el comité de ética del Hospital Ángeles Clínica Londres el 17 de diciembre del 2019. Debido a que el modo de ventilación fue elegido por el médico anestesiólogo tratante de acuerdo con sus habilidades y valoración del paciente 
y que los procedimientos de medición son los requeridos en todo procedimiento anestésico sólo se informó a los pacientes la intensión de uso de sus datos de forma anónima para el protocolo.

\section{Análisis estadístico}

Para llevar a cabo dicha comparación entre las variables, se realizó un análisis de varianza de medidas repetidas $3 \times 3$, el cual permitió identificar las diferencias al interior de cada grupo durante cada fase (intubación, insuflación y resolución) y entre las modalidades de ventilación (VCV, VCP y VCP-GV); así como la posible interacción momento-modo ventilatorio. Sí se demostraron diferencias estadísticamente significativas con un valor de $\mathrm{p}<0.05$ en alguno de estos modelos; se realizó prueba post hoc de Holm por errores en grupos (pool) con términos de factores, corrigiendo una significancia de $\mathrm{p}<0.005$ como significativas para evitar sesgo por análisis múltiple. Los datos fueron vaciados en una base que posteriormente se utilizó para el análisis estadístico. Se utilizó el programa estadístico JASP Team (2020). JASP (Versión 0.14) [Computer software]. Los promedios son referidos como medias con errores típicos. Las variables en escala nominal se obtuvieron con análisis de frecuencia y porcentajes.

\section{RESULTADOS}

Se trabajó con una muestra de 21 pacientes. La comparación entre los grupos se muestra en la Tabla 1. Las principales diferencias

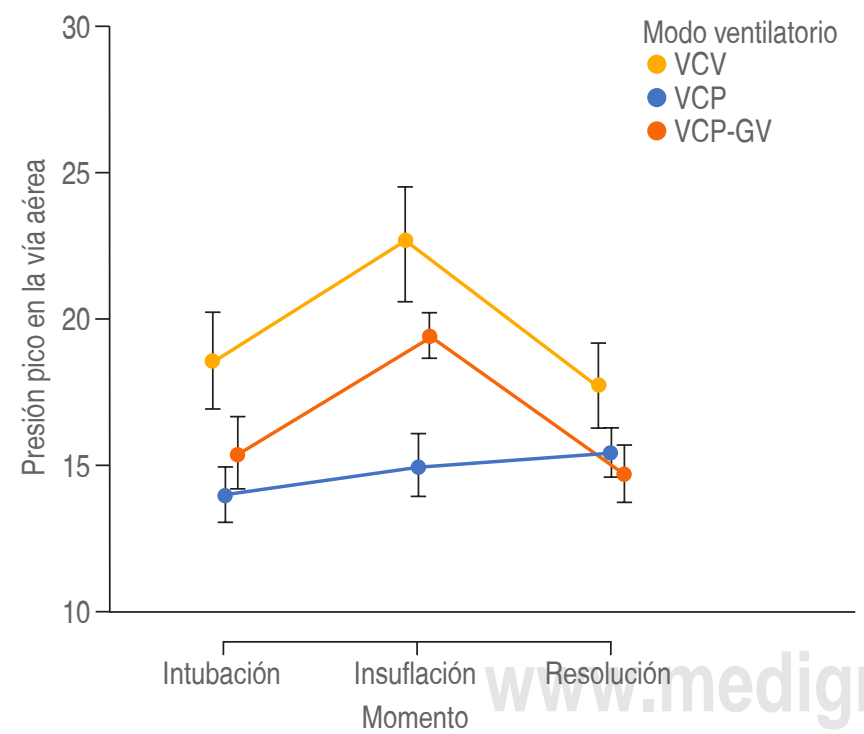

Figura 4: Comportamiento de la presión pico en la vía aérea $\left(\mathrm{cmH}_{2} \mathrm{O}\right)$ en los diferentes momentos de evaluación según el modo de ventilación controlada: por volumen (VCV), por presión (VCP) o por presión con garantía de volumen (VCP-GV). Los puntos indican la media, las barras errores típicos (1ET). Se encontró efecto del momento $(F=22.02, p<0.001)$, por el modo ventilatorio $(F=4.45, p=0.03)$ y por interacción modo-momento $(F=5.5, p<0.0001)$.

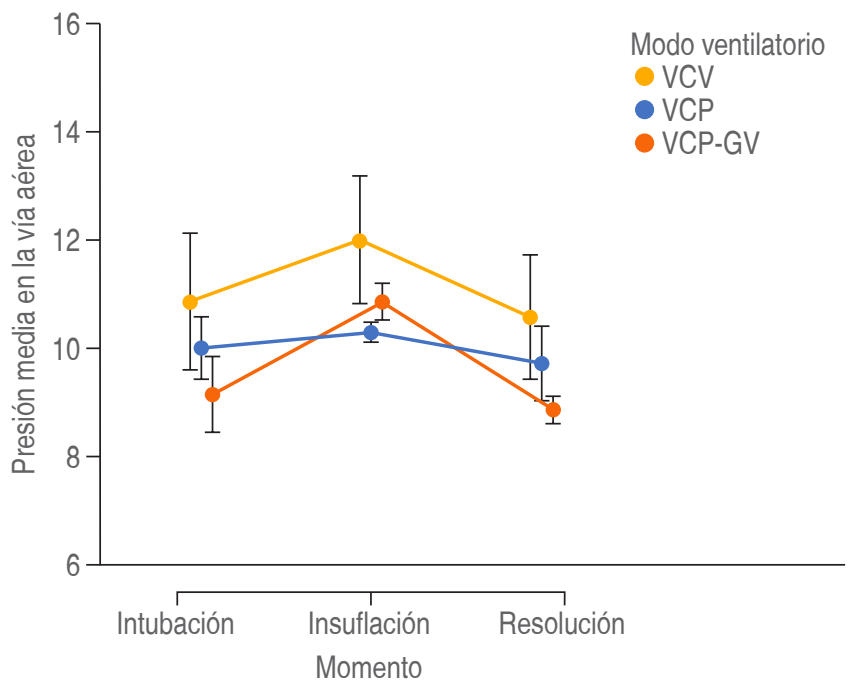

Figura 5: Comportamiento de la presión media $\left(\mathrm{cmH}_{2} \mathrm{O}\right)$ en los diferentes momentos de evaluación según el modo de ventilación controlada: por volumen (VCV), por presión (VCP) o por presión con garantía de volumen (VCP-GV). Los puntos indican la media, las barras errores típicos (1ET). Se encontró efecto del momento $(F=9.85, p<0.001)$, pero no para el modo ventilatorio $(F=1.15, p=0.34) 0$ en la interacción modo-momento $(F=1.15, p=0.35)$.

fueron un mayor predominio de mujeres en el grupo VCP, un IMC más alto en el VCV y más comorbilidades en el grupo VCP-GV. En todos los grupos el procedimiento anestésico y quirúrgico se llevó a cabo sin complicaciones. No tuvimos casos donde el procedimiento laparoscópico se convirtiera en abierto.

\section{Dinámica ventilatoria}

En la frecuencia respiratoria no encontramos diferencias estadísticamente significativas en cuanto al comportamiento de la frecuencia respiratoria entre los grupos y en los diferentes momentos del seguimiento (Figura 1). Sin embargo, el grupo VCP, al momento de la insuflación mostró mayor incremento en su frecuencia respiratoria.

Para el volumen espiratorio se observaron variaciones entre los momentos en los tres grupos, con evidencia de reducción mayor para los grupos VCV y VCP durante la insuflación. El grupo VCP-GV se mantuvo más estable (Figura 2). Al final, en todos los grupos se recuperó el volumen espiratorio a cifras cercanas a lo basal, sin diferencias entre grupos.

Con respecto al dióxido de carbono al final de la espiración $\left(\mathrm{EtCO}_{2}\right)$ durante la anestesia, se mantuvo estable en los tres modos de ventilación (Figura 3).

En la medición de las presiones pico en la vía aérea sí encontramos diferencias según el modo de ventilación. Para los modos VCV y VCP-GV aumentó durante la insuflación con recuperación en la resolución ( $\mathrm{p}<0.001)$. Asimismo, el grupo con VCV en todo momento mostró ser mayor al de 
VCP-GV, aunque esta diferencia no alcanzó la significancia estadística ( $p>0.05)$. Con relación al modo VCP, sus presiones se mantuvieron estables durante los tres momentos de evaluación y sólo fue diferente al modo VCV durante la insuflación $(p=0.01)$. Nuevamente, la resolución en todos los grupos se normalizó la presión pico (Figura 4).

La presión media durante el ciclo respiratorio fue escasamente modificada durante el procedimiento, principalmente durante la insuflación en los grupos de modo VCP y VCP-GV (Figura 5).

En la complianza o distensibilidad pulmonar, como se muestra en la Figura 6, hubo modificaciones durante la insuflación, en la cual se observó reducción en los tres modos de ventilación, para posteriormente recuperarse en la resolución. Entre los grupos no hubo diferencias estadísticamente significativas.

La saturación de oxígeno medida por pulsioximetría evidenció estabilidad en todo el procedimiento y sin diferencias entre las modalidades de ventilación (Figura 7).

Por último, en el seguimiento del volumen minuto no encontramos diferencia estadísticamente significativa según el modo de ventilación, aunque en los pacientes en modo VCV este volumen incrementó más durante la fase de resolución ( $\mathrm{p}=0.01$ ) (Figura 8).

\section{DISCUSIÓN}

Después del análisis se encontró que en todos los grupos existe un incremento de la presión pico como es esperado;

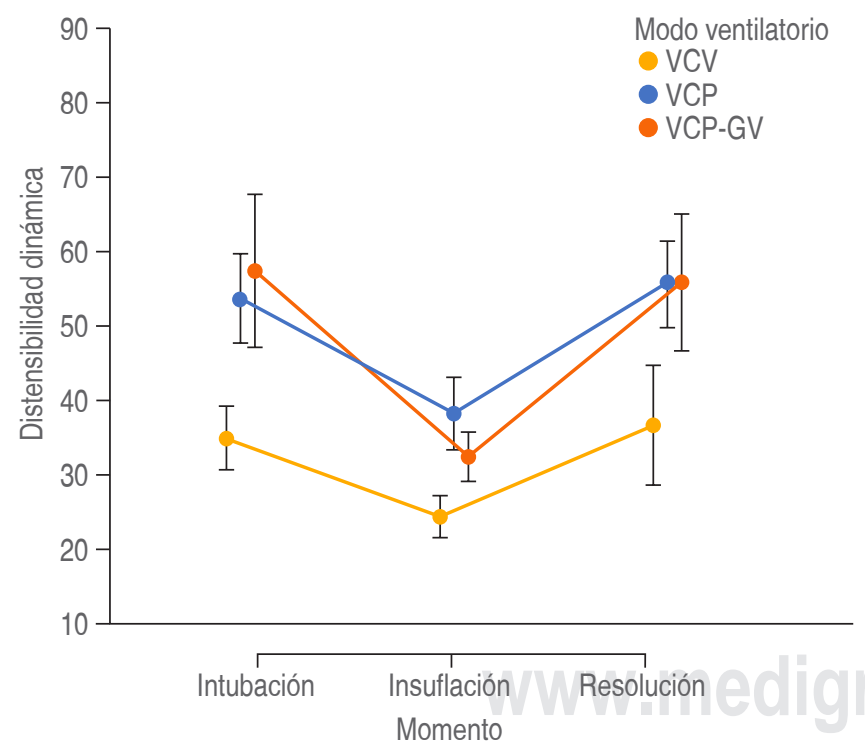

Figura 6: Comportamiento de la distensibilidad $\left(\mathrm{CmH}_{2} \mathrm{O}\right)$ en los diferentes momentos de evaluación según el modo de ventilación controlada: por volumen (VCV), por presión (VCP) o por presión con garantía de volumen (VCP-GV). Los puntos indican la media, las barras errores típicos (1ET). Se encontró efecto del momento $(F=22.3, p<0.001)$, pero no para el modo ventilatorio $(F=2.78, p=0.09)$ o en la interacción modo-momento $(F=1.11, p=0.37)$.

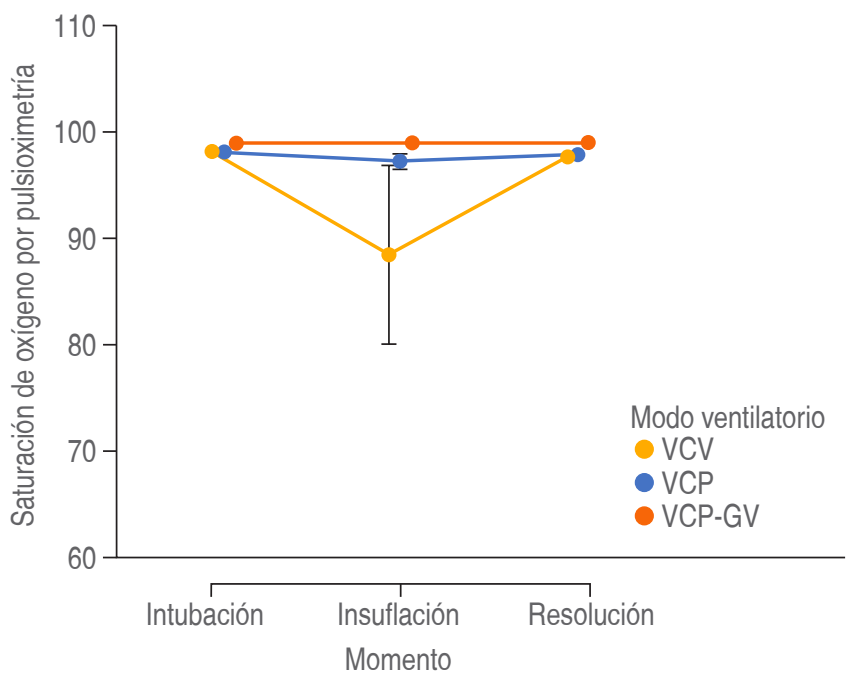

Figura 7: Comportamiento de la saturación de oxígeno por pulsioximetría (\%) en los diferentes momentos de evaluación según el modo de ventilación controlada: por volumen (VCV), por presión (VCP) o por presión con garantía de volumen (VCP-GV). Los puntos indican la media, las barras errores típicos (1ET). No se encontró efecto del momento ( $F=1.5, p=0.24)$, ni para el modo ventilatorio $(F=1.69, p=0.21)$, ni en la interacción modo-momento $(F=1.13, p=0.36)$.

sin embargo, predomina en el modo VCV. La necesidad de aumentar la frecuencia respiratoria se dio en todos los modos, pero sobre todo en el modo VCP.

En los grupos controlados por volumen y por presión se observó una disminución en el volumen corriente espiratorio siendo mayor la diferencia entre el volumen programado y el espiratorio en la fase de insuflación en estos grupos; sin embargo, después no se encontraron diferencias significativas entre los grupos.

Podemos observar que al ser un modo que garantiza una entrega de volumen predeterminado sin permitir la elevación de la presión dentro de la vía aérea al mantener la misma constante ${ }^{(15)}$, el grupo 3 (VCP-GV) mantiene una diferencia menor en esta relación, con lo cual podemos inferir que en el postoperatorio habría una menor incidencia de atelectasias; sin embargo, esto requiere comprobación con un estudio de imagen y tener en cuenta el alto porcentaje de pacientes que las presenta después de una anestesia general. Tampoco se excedieron en ninguna de las fases los $9 \mathrm{~mL} / \mathrm{kg}$, lo cual nos traduce que no ocurrió hiperinsuflación pulmonar en ninguno de los grupos ${ }^{(16)}$.

En cuanto a las presiones dentro de la vía aérea, se observó un aumento en la fase de insuflación en los grupos VCV y VCP-GV en mayor proporción que en el grupo controlado por presión (VCP), en el cual se mantiene más estable respecto a las cifras previas, se descartó cualquier otra situación que pudiera aumentar este parámetro como lo es el broncoespasmo u oclusión de la cánula endotraqueal; sin embargo, en ninguno de los grupos se eleva la presión pico por arriba de $35 \mathrm{mmHg}^{(17)}$, por lo que podemos inferir que hubo un riesgo mínimo de 


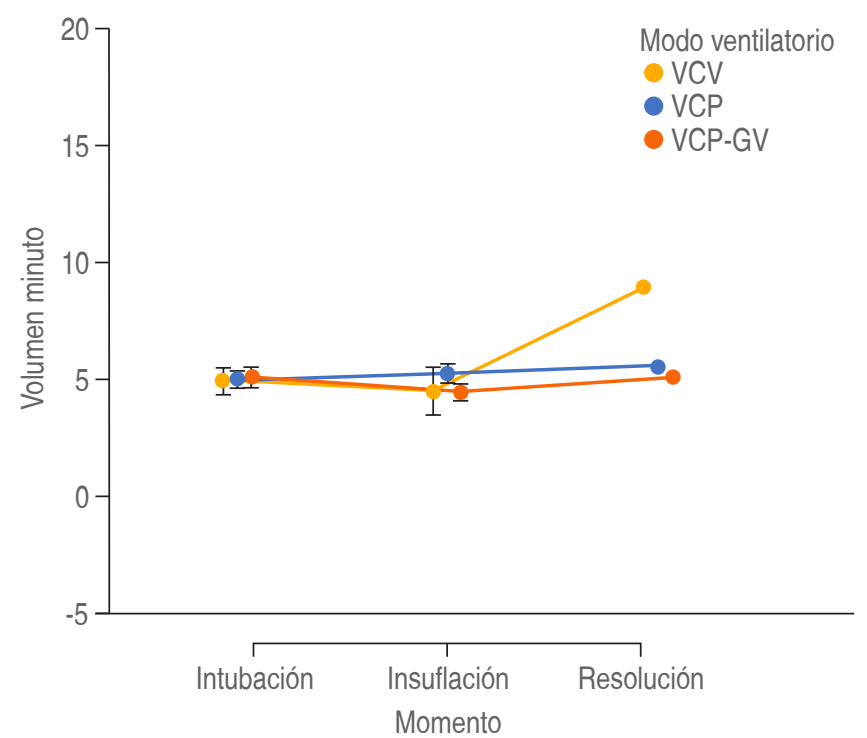

Figura 8: Comportamiento del volumen minuto (L/min) en los diferentes momentos de evaluación según el modo de ventilación controlada: por volumen (VCV), por presión (VCP) o por presión con garantía de volumen (VCP-GV). Los puntos indican la media, las barras errores típicos (1ET). No se encontró efecto del momento ( $F=1.3, p=0.28)$, ni para el modo ventilatorio $(F=0.43, p=0.66)$, ni en la interacción modo-momento ( $F$ $=0.82, p=0.52$. barotrauma, el grupo que mejor mantiene este parámetro con relación a su basal es el grupo controlado por presión, donde ésta se comporta como variable independiente. Debería observarse y compararse este resultado con pacientes cuyas características predispongan al aumento de las presiones como es el caso de obesos o los cambios de posición en el transoperatorio.

La distensibilidad dinámica que depende de la relación presión-volumen no debería ser menor de $40 \mathrm{~mL} / \mathrm{cmH}_{2} \mathrm{O}$ para evitar la aparición de atelectasias y complicaciones en el postoperatorio, aunque se observó una disminución en la misma en los tres grupos, la cual regresa a lo basal o cercano a ésta al término del procedimiento.

Sería necesario correlacionar los datos obtenidos con estudios de imagen o datos obtenidos por gasometría, sería de importancia realizar la comparación bajo otros contextos clínicos que pudieran afectar la presión intraabdominal e intratorácica. Se observó que ningún modo es superior a otro en este estudio.

\section{CONCLUSIONES}

De acuerdo con nuestros datos, las modalidades de ventilación controladas por volumen, por presión o presión con garantía de volumen no parecen diferir en el control de la ventilación en pacientes durante una anestesia para una colecistectomía laparoscópica, si son utilizadas de acuerdo con las necesidades del paciente.

\section{REFERENCIAS}

1. Ball L, Costantino F, Fiorito M, Amodio S, Pelosi P. Respiratory mechanics during general anaesthesia. Ann Transl Med. 2018;6:379.

2. Atkinson TM, Giraud GD, Togioka BM, Jones DB, Cigarroa JE. Cardiovascular and ventilatory consequences of laparoscopic surgery. Circulation. 2017;135:700-710.

3. Hedenstierna G, Edmark L. Mechanisms of atelectasis in the perioperative period. Best Pract Res Clin Anaesthesiol. 2010;24:157-169.

4. Dresse C, Joris JL, Hans GA. Mechanical ventilation during anaesthesia: pathophysiology and clinical implications. Trends Anaesth Crit Care. 2012;2:71-75.

5. Tobin MJ. Physiologic basis of mechanical ventilation. Ann Am Thorac Soc. 2018;15:S49-S52.

6. Hallett S, Ashurst JV. Physiology, tidal volume. [Updated 2019 Feb 2]. In: StatPearls [Internet]. Treasure Island (FL): StatPearls Publishing; 2019. Available in: https://www.ncbi.nlm.nih.gov/books/NBK482502/

7. Kim MS, Soh S, Kim SY, Song MS, Park JH. Comparisons of pressurecontrolled ventilation with volume guarantee and volume-controlled 1:1 equal ratio ventilation on oxygenation and respiratory mechanics during robot-assisted laparoscopic radical prostatectomy: a randomizedcontrolled trial. Int J Med Sci. 2018;15:1522-1529.

8. Assad OM, El Sayed AA, Khalil MA. Comparison of volume-controlled ventilation and pressure-controlled ventilation volume guaranteed during laparoscopic surgery in Trendelenburg position. J Clin Anesth. 2016;34:55-61.

9. Aydin V, Kabukcu HK, Sahin N, Mesci A, Arici AG, Kahveci G, et al. Comparison of pressure and volume-controlled ventilation in laparoscopic cholecystectomy operations. Clin Respir J. 2016;10:342-349.
10. Mihalj M, Vladic D, Karlovic Z, Zadro Z, Majeric Kogler V. Effects of pressure-controlleda volume-controlled ventilation on hemodynamic and respiratory parameters in patients during laparoscopic cholecystectomy. Acta Clin Croat. 2017;56:555-560.

11. Movassagi R, Montazer M, Mahmoodpoor A, Fattahi V, Iranpour A, Sanaie S. Comparison of pressure vs. volume controlled ventilation on oxygenation parameters of obese patients undergoing laparoscopic cholecystectomy. Pak J Med Sci. 2017;33:1117-1122.

12. Diaz R, Heller D. Barotrauma and mechanical ventilation. [Updated 2019 Oct 1]. In: StatPearls [Internet]. Treasure Island (FL): StatPearls Publishing; 2019. Available in: https://www.ncbi. nlm.nih.gov/books/NBK545226/ Mechanisms of atelectasis in the perioperative period.

13. Jo YY, Kwak HJ. What is the proper ventilation strategy during laparoscopic surgery? Korean J Anesthesiol. 2017;70:596-600.

14. Martin DC, Richards GN. Predicted body weight relationships for protective ventilation - unisex proposals from pre-term through to adult. BMC Pulm Med. 2017;17:85.

15. Ball L, Dameri M, Pelosi P. Modes of mechanical ventilation for the operating room. Best Pract Res Clin Anaesthesiol. 2015;29:285 $\square 299$. doi: 10.1016/j.bpa.2015.08.003.

16. Gammon RB, Shin MS, Buchalter SE. Pulmonary barotrauma in mechanical ventilation. Patterns and risk factors. Chest. 1992;102:568-572.

17. Grieco DL, Russo A, Romano B, Anzellotti GM, Ciocchetti P, Torrini F, et al. Lung volumes, respiratory mechanics and dynamic strain during general anaesthesia. Br J Anaesth. 2018;121:1156-1165. 